\title{
The role of symplectic integrators in optimal control
}

\author{
Monique Chyba ${ }^{1 *}$, Ernst Hairer ${ }^{2}$ and Gilles Vilmart ${ }^{2,3}$ \\ 1 University of Hawaii, Department of Mathematics, 2565 Mc Carthy the Mall, Honolulu 96822 \\ 2 Université de Genève, Section de mathématiques, 2-4 rue du Lièvre, CH-1211 Genève 4, Switzerland \\ 3 INRIA Rennes, ENS Cachan Antenne de Bretagne, Avenue Robert Schumann, 35170 Bruz, France
}

\begin{abstract}
SUMMARY
For general optimal control problems, Pontryagin's maximum principle gives necessary optimality conditions which are in the form of a Hamiltonian differential equation. For its numerical integration, symplectic methods are a natural choice. This article investigates to which extent the excellent performance of symplectic integrators for long-time integrations in astronomy and molecular dynamics carries over to problems in optimal control.

Numerical experiments supported by a backward error analysis show that, for problems in low dimension close to a critical value of the Hamiltonian, symplectic integrators have a clear advantage. This is illustrated using the Martinet case in sub-Riemannian geometry. For problems like the orbital transfer of a spacecraft or the control of a submerged rigid body such an advantage cannot be observed. The Hamiltonian system is a boundary value problem and the time interval is in general not large enough so that symplectic integrators could benefit from their structure preservation of the flow. Copyright (c) 2002 John Wiley \& Sons, Ltd.
\end{abstract}

KEY WORDS: symplectic integrator, backward error analysis, sub-Riemannian geometry, Martinet, abnormal geodesic, orbital transfer, submerged rigid body.

\section{INTRODUCTION}

For the numerical solution of optimal control problems there are essentially two approaches: the direct approach which consists in discretizing the problem directly and applying optimization techniques, and the so-called indirect approach which is based on Pontryagin's maximum principle. This gives necessary conditions that reduce the optimal control problem to a system of Hamiltonian differential equations with boundary conditions, which can be solved by shooting techniques. The present article is concerned with the indirect approach.

There are many arguments in favour of using symplectic integrators for the numerical solution of the Hamiltonian system. Firstly, geometric numerical integration [1] puts forward

\footnotetext{
*Correspondence to: mchyba@math.hawaii.edu

Contract/grant sponsor: This work was partially supported by the Fonds National Suisse, project No. 200020109158 and by the National Science Foundation grant DMS-030641.
} 
the use of structure-preserving algorithms for the solution of structured problems like Hamiltonian systems. This is justified by a backward error analysis which allows one to interpret the numerical solution of a symplectic method as the exact flow of a modified Hamiltonian system. This explains the excellent long-time behavior of such integrators. Furthermore, a series of papers $[2,3,4,5]$ develops variational integrators for optimal control problems and emphasizes their symplecticity. The work of $[6,7]$ shows that, for partitioned Runge-Kutta discretizations based on symplectic pairs, the direct and indirect approaches are equivalent.

On the other hand, the Hamiltonian systems arising in optimal control are quite different from those in astronomy and molecular dynamics, where symplectic integrators have proven to be the method of choice. Pontryagin's maximum principle yields a boundary value problem and long-time integration is in general not an issue. Furthermore, the modified Hamiltonian system (in the sense of backward error analysis) is not necessarily a differential equation that arises from a modified optimal control problem. It is therefore not obvious whether symplectic integrators will be superior to standard methods. The aim of this article is to study this question and to investigate the practical effect of using symplectic integrators in the numerical solution of optimal control problems. This will be done at several case studies.

For problems in low dimension which are close to a critical value of the Hamiltonian, symplectic integrators turn out to have a significant advantage. This happens for the socalled Martinet case in sub-Riemannian geometry, see $[8,9]$ and the references therein. The Martinet flat case and a non integrable perturbation are introduced in Sect. 2 together with the corresponding differential equations. Numerical experiments with an explicit Runge-Kutta method and with the symplectic Störmer-Verlet method are presented in Sect. 3 and illustrated with figures. Close to abnormal geodesics, the results are quite spectacular. For a relatively large step size, the symplectic integrator provides a solution with the correct qualitative behavior and a satisfactory accuracy, while for the same step size the non-symplectic integrator gives a completely wrong numerical solution, particularly for the non integrable case. The explanation relies on the theory of backward error analysis (Sect.4).

Unfortunately, this explanation cannot be extended to general optimal control problems. We present two examples: the orbital transfer of a spacecraft (Sect.5) and the control of a submerged rigid body (Sect. 6). The Hamiltonian system for the submerged rigid body is very sentitive when considered as an initial value problem and thus requires the use of multiple shooting for solving the boundary value problem. For both problems, symplectic integrators do not show any real advantage. The reason is that the time interval is not long enough so that the symplectic integrator could benefit from structure preservation.

\section{A MARTINET TYPE SUB-RIEMANNIAN STRUCTURE}

Let $(U, \Delta, g)$ be a sub-Riemannian structure where $U$ is an open neighborhood of $R^{3}, \Delta$ a distribution of constant rank 2 and $g$ a Riemannian metric. When $\Delta$ is a contact distribution, there are no abnormal geodesics, and a non-symplectic integrator is as efficient as a symplectic one. However, when the distribution is taken as the kernel of the Martinet one-form, we show that a symplectic integrator is much more efficient for the computation of the normal geodesics and their conjugate points near the abnormal directions.

We briefly recall some results of [8] for a sub-Riemannian structure $(U, \Delta, g)$. Here, $U$ is an 
open neighborhood of the origin in $R^{3}$ with coordinates $q=(x, y, z)$, and $g$ is a Riemannian metric for which a graduated normal form, at order 0 , is $g=(1+\alpha y) d x^{2}+(1+\beta x+\gamma y) d y^{2}$. The distribution $\Delta$ is generated by the two vector fields $F_{1}=\frac{\partial}{\partial x}+\frac{y^{2}}{2} \frac{\partial}{\partial z}$ and $F_{2}=\frac{\partial}{\partial y}$ which correspond to $\Delta=\operatorname{ker} \omega$ where $\omega=d z-\frac{y^{2}}{2} d x$ is the Martinet canonical one-form. To this distribution we associate the affine control system

$$
\dot{q}=u_{1}(t) F_{1}(q)+u_{2}(t) F_{2}(q),
$$

where $u_{1}(t), u_{2}(t)$ are measurable bounded functions which act as controls.

We consider two cases, the Martinet flat case $g=d x^{2}+d y^{2}$, an integrable situation, and a one parameter perturbation $g=d x^{2}+(1+\beta x)^{2} d y^{2}$ for which the set of geodesics is non integrable.

\subsection{Geodesics}

It follows from the Pontryagin maximum principle, see $[8,9]$, that the normal geodesics corresponding to $g=d x^{2}+(1+\beta x)^{2} d y^{2}$ are solutions of an Hamiltonian system

$$
\dot{q}=\frac{\partial H}{\partial p}(q, p), \quad \dot{p}=-\frac{\partial H}{\partial q}(q, p),
$$

where $q=(x, y, z)$ is the state, $p=\left(p_{x}, p_{y}, p_{z}\right)$ is the adjoint state, and the Hamiltonian is

$$
H(q, p)=\frac{1}{2}\left(\left(p_{x}+p_{z} \frac{y^{2}}{2}\right)^{2}+\frac{p_{y}^{2}}{(1+\beta x)^{2}}\right) .
$$

In other words, the normal geodesics are solutions of the following equations:

$$
\begin{aligned}
\dot{x} & =p_{x}+p_{z} \frac{y^{2}}{2} & \dot{p}_{x} & =\frac{\beta p_{y}^{2}}{(1+\beta x)^{3}} \\
\dot{y} & =\frac{p_{y}}{(1+\beta x)^{2}} & \dot{p}_{y} & =-\left(p_{x}+p_{z} \frac{y^{2}}{2}\right) p_{z} y \\
\dot{z} & =\left(p_{x}+p_{z} \frac{y^{2}}{2}\right) \frac{y^{2}}{2} & \dot{p}_{z} & =0 .
\end{aligned}
$$

Notice that the variables $z$ and $p_{z}$ do not influence the other equations (except via the initial value $p_{z}(0)$ ), so that we are actually confronted with a Hamiltonian system in dimension four. For the Martinet flat case $(\beta=0)$, the interesting dynamics takes place in the two-dimensional space of coordinates $\left(y, p_{y}\right)$. The Hamiltonian is

$$
H\left(y, p_{y}\right)=\frac{p_{y}^{2}}{2}+\frac{1}{2}\left(p_{x}+p_{z} \frac{y^{2}}{2}\right)^{2},
$$

where $p_{x}$ and $p_{z}$ have to be considered as constants. This is a one-degree of freedom mechanical system with a quartic potential. For $p_{x}<0<p_{z}$, the Hamiltonian $H\left(y, p_{y}\right)$ has two local minima at $\left(y= \pm \sqrt{-2 p_{x} / p_{z}}, p_{y}=0\right)$, which correspond to stationary points of the vector field. In this case, the origin $\left(y=0, p_{y}=0\right)$ is a saddle point.

Whereas normal geodesics correspond to oscillating motion, it is shown in $[8,9]$ that the abnormal geodesics are the lines $z=z_{0}$ contained in the plane $y=0$. For the considered 
metrics, the abnormal geodesics can be obtained as projections of normal geodesics, we say that they are not strictly abnormal. In [9], the authors introduce a geometric framework to analyze the singularities of the sphere in the abnormal direction when $\beta \neq 0$. See also $[10,11]$ for a precise description of the role of the abnormal geodesics in sub-Riemannian geometry in the general non-integrable case, i.e., when the abnormal geodesics can be strict. The major result of these papers is the proof that the sub-Riemannian sphere is not sub-analytic because of the abnormal geodesics.

Interesting phenomena arise when the normal geodesics are close to the separatrices connecting the saddle point. Therefore, we shall consider in Sect. 3 the computation of normal geodesics with $y(0)=0$ and $p_{y}(0)>0$ but small.

\subsection{Conjugate points}

For the Hamiltonian system (1) we consider the exponential mapping

$$
\exp _{q_{0}, t}: p_{0} \longmapsto q\left(t, q_{0}, p_{0}\right)
$$

which, for fixed $q_{0} \in R^{3}$, is the projection $q\left(t, q_{0}, p_{0}\right)$ onto the state space of the solution of (1) starting at $t=0$ from $\left(q_{0}, p_{0}\right)$. Following the definition in [8] we say that the point $q_{1}$ is conjugate to $q_{0}$ along $q(t)$ if there exists $\left(p_{0}, t_{1}\right), t_{1}>0$, such that $q(t)=\exp _{q_{0}, t}\left(p_{0}\right)$ with $q_{1}=\exp _{q_{0}, t_{1}}\left(p_{0}\right)$, and the mapping $\exp _{q_{0}, t_{1}}$ is not an immersion at $p_{0}$. We say that $q_{1}$ is the first conjugate point if $t_{1}$ is minimal. First conjugate points play a major role when studying optimal control problems since it is a well known result that a geodesic is not optimal beyond the first conjugate point.

For the numerical computation of the first conjugate point, we compute the solution of the Hamiltonian system (1) together with its variational equation,

$$
\dot{y}=J^{-1} \nabla H(y), \quad \dot{\Psi}=J^{-1} \nabla^{2} H(y) \Psi .
$$

Here, $y=(q, p)$ and $J$ is the canonical matrix for Hamiltonian systems. It can be shown that for Runge-Kutta methods, the derivative of the numerical solution with respect to the initial value, $\Psi_{n}=\partial y_{n} / \partial y_{0}$, is the result of the same numerical integrator applied to the augmented system (3), see [1, Lemma VI.4.1]. Here, the matrix

$$
\Psi=\left(\begin{array}{ll}
\partial q / \partial q_{0} & \partial q / \partial p_{0} \\
\partial p / \partial q_{0} & \partial p / \partial p_{0}
\end{array}\right)
$$

has dimension $6 \times 6$. The conjugate points are obtained when $\partial q / \partial p_{0}$ becomes singular, i.e., $\operatorname{det}\left(\partial q / \partial p_{0}\right)=0$.

\section{COMPARISON OF SYMPLECTIC AND NON-SYMPLECTIC INTEGRATORS}

For the numerical integration of the Hamiltonian system $(1)$, where we rewrite $\frac{\partial H}{\partial q}(q, p)=$ $H_{q}(q, p)$ and $\frac{\partial H}{\partial p}(q, p)=H_{p}(q, p)$, we consider two integrators of the same order 2 :

- a non-symplectic, explicit Runge-Kutta discretization, denoted RK2 (see [1, Sect. II.1.1]),

$$
\begin{aligned}
q_{n+1 / 2} & =q_{n}+\frac{h}{2} H_{p}\left(q_{n}, p_{n}\right) & p_{n+1 / 2} & =p_{n}-\frac{h}{2} H_{q}\left(q_{n}, p_{n}\right) \\
q_{n+1} & =q_{n}+h H_{p}\left(q_{n+1 / 2}, p_{n+1 / 2}\right) & p_{n+1} & =p_{n}-h H_{q}\left(q_{n+1 / 2}, p_{n+1 / 2}\right)
\end{aligned}
$$


- the symplectic Störmer-Verlet scheme (see e.g. [1, Sect. VI.3]),

$$
\begin{aligned}
p_{n+1 / 2} & =p_{n}-\frac{h}{2} H_{q}\left(q_{n}, p_{n+1 / 2}\right) \\
q_{n+1} & =q_{n}+\frac{h}{2}\left(H_{p}\left(q_{n}, p_{n+1 / 2}\right)+H_{p}\left(q_{n+1}, p_{n+1 / 2}\right)\right) \\
p_{n+1} & =p_{n+1 / 2}-\frac{h}{2} H_{q}\left(q_{n+1}, p_{n+1 / 2}\right)
\end{aligned}
$$

where $q_{n}=\left(x_{n}, y_{n}, z_{n}\right)$ and $p_{n}=\left(p_{x, n}, p_{y, n}, p_{z, n}\right)$. Here, $q_{n} \approx q(n h), p_{n} \approx p(n h)$ and $h$ is the step size.

For the computation of the conjugate points, we apply the numerical methods to the variational equation (3). Notice that only the partial derivatives with respect to $p_{0}$ have to be computed. Conjugate points are then detected when $\operatorname{det}\left(\partial q_{n} / \partial p_{0}\right)$ changes sign. We approximate them by linear interpolation which introduces an error of size $\mathcal{O}\left(h^{2}\right)$. This is comparable to the accuracy of the chosen integrators which are both of second order.
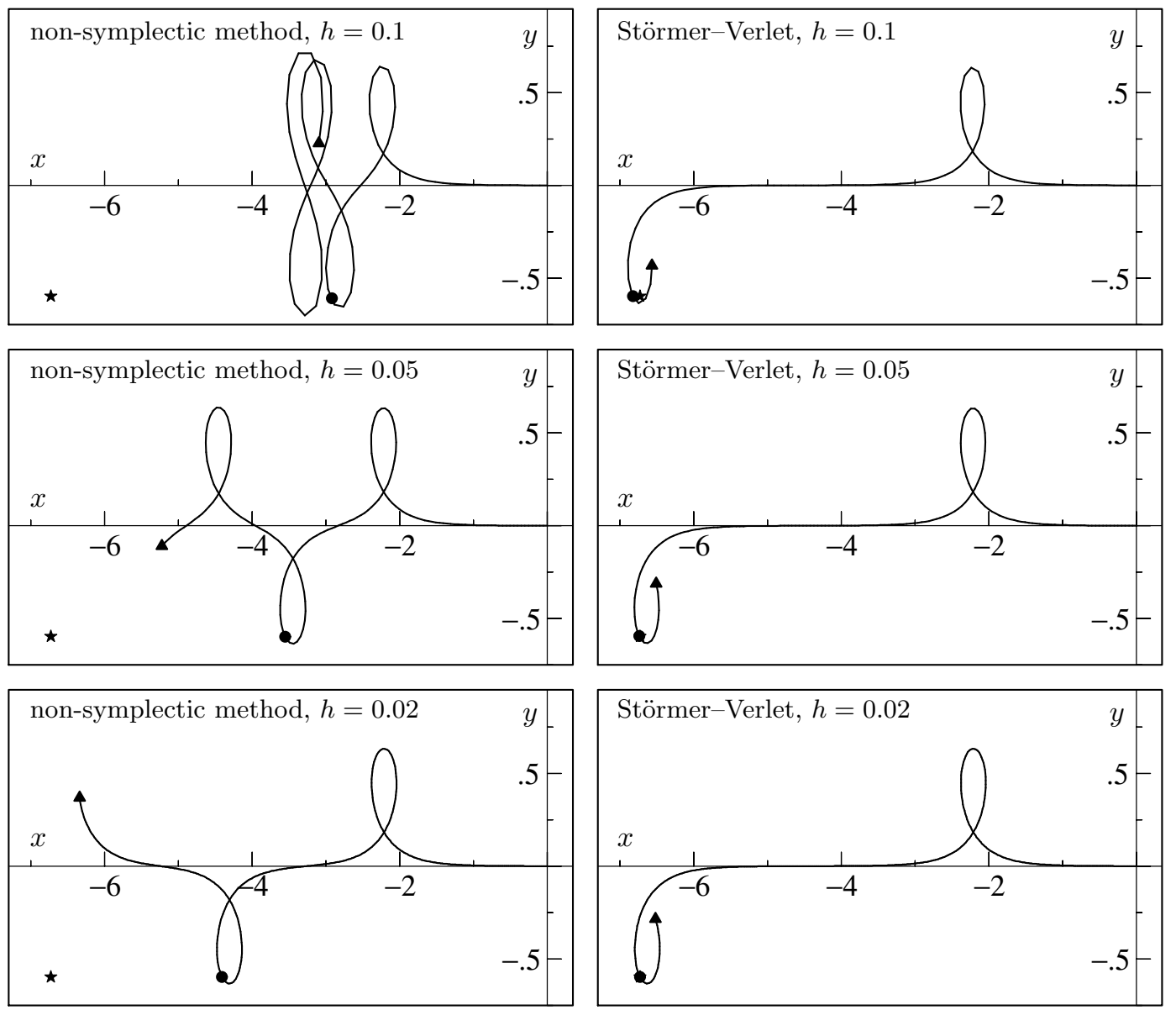

Figure 1. Trajectories in the $(x, y)$-plane for the flat case $\beta=0$.

Copyright (c) 2002 John Wiley \& Sons, Ltd.

Optim. Control Appl. Meth. 2002; 00:1-6 Prepared using ocaauth.cls 
Remark

The Störmer-Verlet scheme (5) is implicit in general. A few fixed point iterations yield the numerical solution with the desired accuracy. Notice however that the method becomes explicit in the Martinet flat case $\beta=0$. One simply has to compute the components in a suitable order, for instance $p_{x, n+1}, p_{z, n+1}, p_{y, n+1 / 2}, y_{n+1}, x_{n+1}, z_{n+1}, p_{y, n+1}$.

\subsection{Martinet flat case}

We consider first the flat case $\beta=0$ in the Hamiltonian system (2). As initial values we choose (cf. [8])

$x(0)=y(0)=z(0)=0, \quad p_{x}(0)=\cos \theta_{0}, \quad p_{y}(0)=\sin \theta_{0}, \quad p_{z}(0)=10, \quad$ where $\quad \theta_{0}=\pi-10^{-3}$,

so that we start close to an abnormal geodesics, and we integrate the system over the interval $[0,9]$.

Figure 1 displays the projection onto the $(x, y)$-plane of the numerical solution obtained with different step sizes $h$ by the two integrators. The initial value is at the origin, and the final state is indicated by a triangle. The circles represent the first conjugate point detected along the numerical solution, while the stars give the position of the first conjugate point on the exact solution of the problem. There is an enormous difference between the two numerical integrators. The symplectic (Störmer-Verlet) method (5) provides a qualitatively correct solution already with a large step size $h=0.1$, and it gives an excellent approximation for step sizes smaller than $h=0.05$. On the other hand, the non-symplectic, explicit Runge-Kutta method (4) gives completely wrong results, and step sizes smaller than $10^{-3}$ are needed to provide an acceptable solution. An explanation of the different behavior of the two integrators will be given in Sect. 4 below.
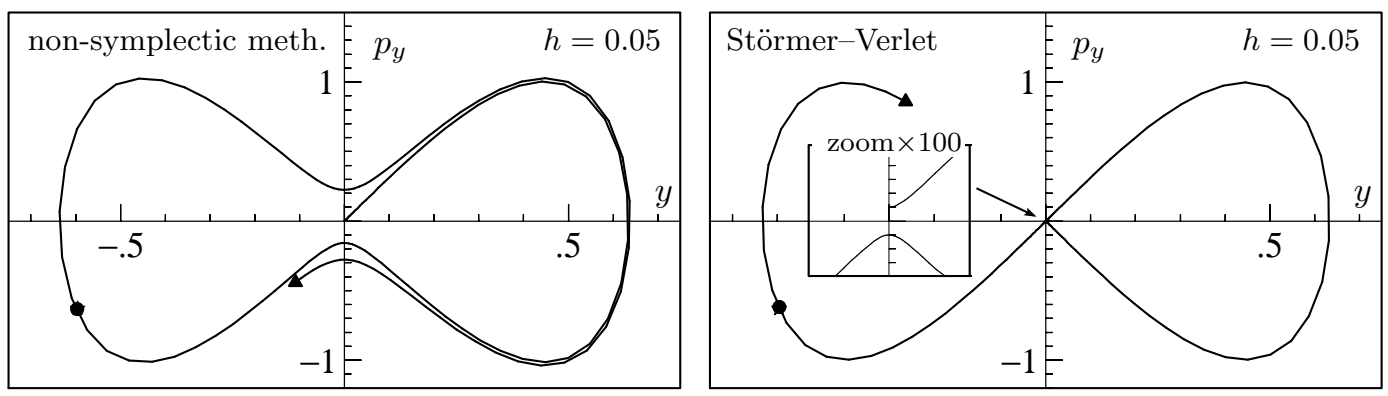

Figure 2. Phase portraits in the $\left(y, p_{y}\right)$-plane for the flat case $\beta=0$.

As noticed in Sect. 2, the normal geodesics in the flat case are determined by a one-degree of freedom Hamiltonian system in the variables $y$ and $p_{y}$. We therefore show in Figure 2 the projection onto the $\left(y, p_{y}\right)$-space of the solutions previously computed with step size $h=0.05$. The exact solution starts at $\left(0, \sin \theta_{0}\right)$ above the saddle point, turns around the positive stationary point, crosses the $p_{y}$-axis at $\left(0,-\sin \theta_{0}\right)$, turns around the negative stationary point, and then continues periodically. The numerical approximation by the non-symplectic method covers more than one and a half periods, whereas the Störmer-Verlet and the exact solution cover less than one period for the time interval $[0,9]$. Since the conjugate point is not very sensible with respect to perturbations in the initial value for $p_{y}$, the $\left(y, p_{y}\right)$ coordinates of 
Table I. Accuracy for the first conjugate time.

\begin{tabular}{|c|c|c|}
\hline \multicolumn{3}{|c|}{ Martinet flat case } \\
\hline$h$ & RK2 & Verlet \\
\hline $10^{-1}$ & 4.504945 & $\underline{8.504716}$ \\
$10^{-2}$ & 6.748262 & $\underline{8.416622}$ \\
$10^{-3}$ & $\underline{8.360340}$ & $\underline{8.4164} 12$ \\
$10^{-4}$ & $\underline{8.416} 349$ & $\underline{8.416410}$ \\
\hline \multicolumn{3}{|c|}{ exact solution: $t_{1} \approx \underline{8.416409}$} \\
\hline
\end{tabular}

\begin{tabular}{|c|c|c|}
\hline \multicolumn{3}{|c|}{ Non integrable situation } \\
\hline$h$ & RK2 & Verlet \\
\hline $10^{-1}$ & 4.511294 & $\underline{4.883832}$ \\
$10^{-2}$ & 7.380322 & $\underline{4.877056}$ \\
$10^{-3}$ & $\underline{4.877183}$ & $\underline{4.876998}$ \\
$10^{-4}$ & $\underline{4.876997}$ & $\underline{4.876997}$ \\
\hline \multicolumn{3}{|c|}{ exact solution: $t_{1} \approx \underline{4.876997}$} \\
\hline
\end{tabular}

the conjugate point obtained by the non-symplectic integrator are rather accurate, but the corresponding integration time is completely wrong.

Table I lists the conjugate time obtained with the two integrators using various step sizes. There is a significant difference between the two methods. We can see that with the StörmerVerlet method (5) a step size of order $h=10^{-2}$ provides a solution with 4 correct digits. A step size a 100 times smaller is needed to get the same precision with the non-symplectic method.

\subsection{Non integrable perturbation}

For our next numerical experiment we choose the perturbation parameter $\beta=-10^{-4}$ in the differential equation (2). We consider the same initial values and the same integration interval as in Sect. 3.1. The exact solution is no longer periodic and, due to the fact that $\beta$ is chosen negative, its projection onto the $\left(y, p_{y}\right)$-space slowly spirals inwards around the positive stationary point (see right picture in Figure 4).
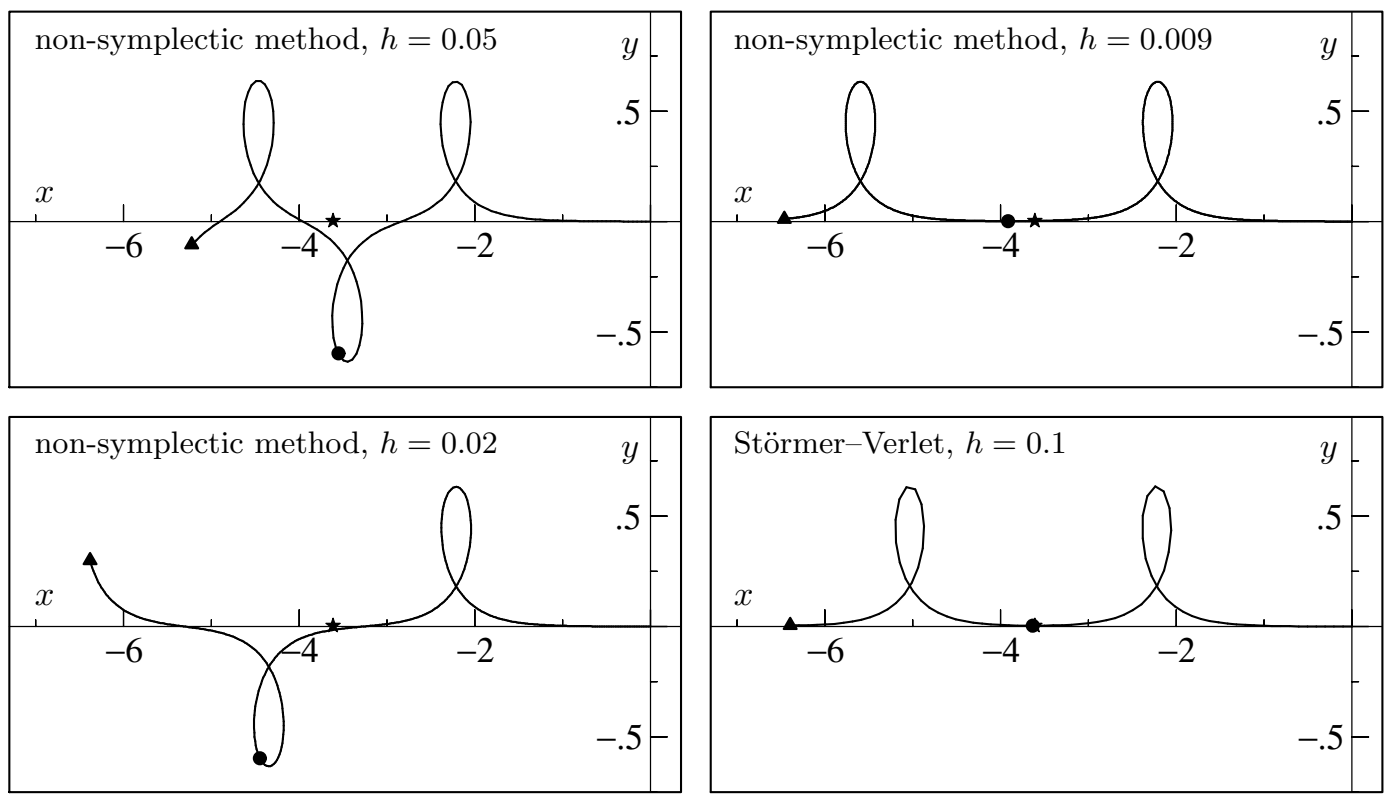

Figure 3. Trajectories in the $(x, y)$-plane for the non integrable case $\beta=-10^{-4}$. 

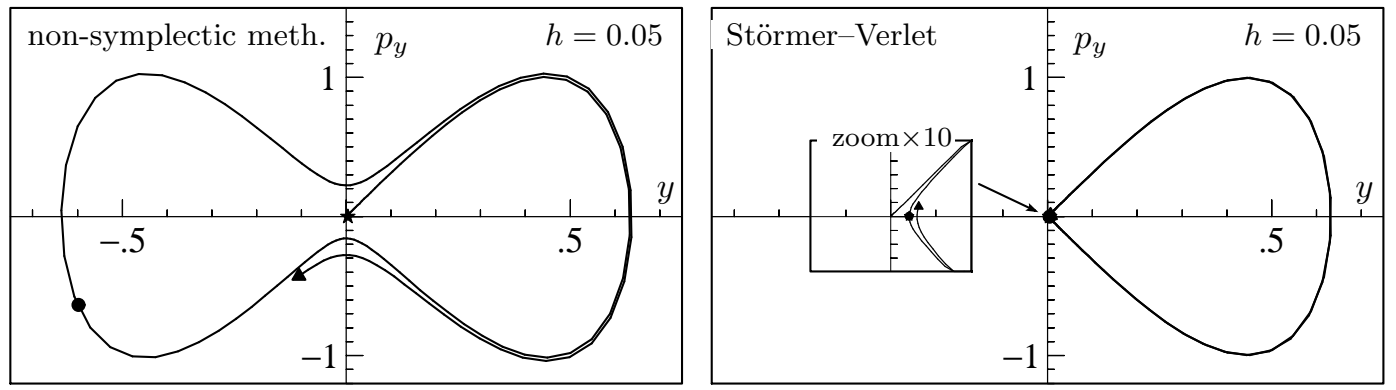

Figure 4. Phase portraits in the $\left(y, p_{y}\right)$-plane for the non integrable case $\beta=-10^{-4}$.

Figures 3 and 4 and Table I display the numerical results obtained by the two integrators for the differential equation (2) with $\beta=-10^{-4}$. The interpretation of the symbols (triangles, circles, and stars) is the same as before. The excellent behavior of the symplectic integrator is even more spectacular than in the flat case, and the pictures obtained for the StörmerVerlet method agree extremely well with the exact solution. The non-symplectic method gives qualitatively wrong solutions for step sizes larger than $h=0.01$. In the $\left(y, p_{y}\right)$-space it alternatively spirals around the right and left stationary points whereas the exact solution spirals only around the positive stationary point. In contrast to the Martinet flat case, the conjugate point obtained by the non-symplectic method is here wrong also in the $\left(y, p_{y}\right)$-space.

\subsection{An asymptotic formula on the first conjugate time in the Martinet flat case}

Now that we have shown the efficiency of symplectic integrators, we can make more precise the asymptotic behavior studied in [8]. For the initial values of (6) and $\beta=0$, consider the ratio $R=t_{1} \sqrt{p_{z}} /(3 K(k))$ where $t_{1}$ is the first conjugate time for the normal geodesic, and $K(k)$ is an elliptic integral of the first kind,

$$
K(k)=\int_{0}^{\pi / 2} \frac{1}{\sqrt{1-k^{2} \sin ^{2} u}} d u, \quad k=\sin \left(\theta_{0} / 2\right) .
$$

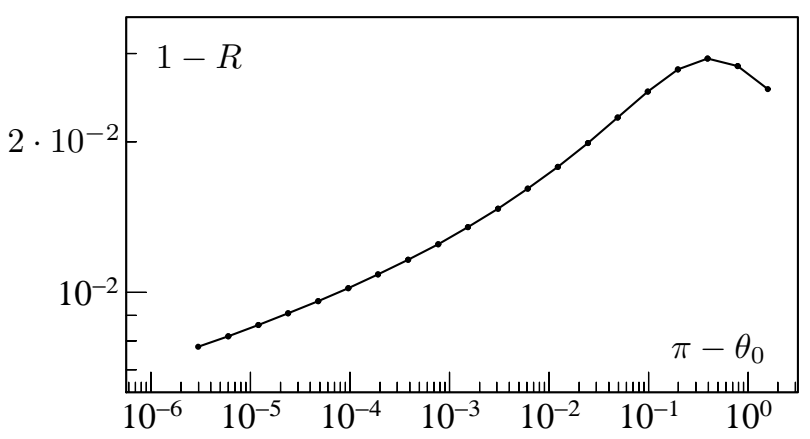

Figure 5. Illustration of the asymptotic behavior of $R$ (StörmerVerlet scheme with step size $h=10^{-4}$ ). 
By studying analytic solutions for the normal geodesics, it is proved in [8] that this ratio satisfies the inequality $2 / 3 \leq R \leq 1$. It follows from a rescaling of the equations (2) that $R$ is independent of $p_{z}$.

In Figure 5, we represent the values of $1-R$ as a function of $\varepsilon=\pi-\theta_{0}$, for various initial values $\theta_{0}$. The numerical results indicate that the ratio $R$ depends on $\theta_{0}$, and $R \longrightarrow 1^{-}$slowly for $\theta_{0} \longrightarrow \pi^{-}$.

\section{BACKWARD ERROR ANALYSIS}

The theory of backward error analysis is fundamental for the study of geometric integrators and it is treated in much detail in the monographs of Sanz-Serna \& Calvo [12], Hairer, Lubich \& Wanner [1, Chap. IX], and Leimkuhler \& Reich [13]. It allows us to explain the numerical phenomena encountered in the previous section.

\subsection{Backward error analysis and energy conservation}

We briefly present the main ideas of backward error analysis for the study of symplectic integrators, see [1, Chap. IX]. Consider a system of differential equations

$$
\dot{y}=f(y), \quad y(0)=y_{0}
$$

and a numerical integrator $y_{n+1}=\Phi_{h}\left(y_{n}\right)$ of order $p$. The idea is to search for a modified differential equation written as a formal series in powers of the step size $h$,

$$
\dot{\tilde{y}}=\widetilde{f}(\widetilde{y})=f(\widetilde{y})+h^{p} f_{p+1}(\widetilde{y})+h^{p+1} f_{p+2}(\widetilde{y})+\ldots,
$$

such that $y_{n}=\widetilde{y}\left(t_{n}\right)$ for $t_{n}=n h, n=0,1,2, \ldots$, in the sense of formal power series. The motivation of this approach is that it is often easier to study the modified equation (8) than directly the numerical solution.

What makes backward error analysis so important for the study of symplectic integrators is the fact that, when applied to a Hamiltonian system $\dot{y}=J^{-1} \nabla H(y)$, the modified equation (8) has the same structure $\dot{\widetilde{y}}=J^{-1} \nabla \widetilde{H}(\widetilde{y})$ with a modified Hamiltonian

$$
\widetilde{H}(y)=H(y)+h^{p} H_{p+1}(y)+h^{p+1} H_{p+2}(y)+\ldots .
$$

However, the series usually diverges, so a truncation at a suitable order $N(h)$ is necessary,

$$
\widetilde{H}(y)=H(y)+h^{p} H_{p+1}(y)+\ldots+h^{N-1} H_{N}(y) .
$$

This truncation induces an error that can be made exponentially small, by choosing $N(h) \sim$ $C / h$, see [1, Theorem IX.8.1]. More precisely, we have that for $t_{n}=n h$ and $h \rightarrow 0$,

$$
\widetilde{H}\left(y_{n}\right)=\widetilde{H}\left(y_{0}\right)+\mathcal{O}\left(t_{n} e^{-h_{0} / h}\right) .
$$

as long as the numerical solution $\left\{y_{n}\right\}$ stays in a compact set. On intervals of length $\mathcal{O}\left(e^{h_{0} / 2 h}\right)$, the modified Hamiltonian $\widetilde{H}(y)$ is thus exactly conserved up to exponentially small terms. 


\subsection{Backward error analysis for the Martinet problem}

Symplectic integrators are successfully applied in the long-time integration of Hamiltonian systems, for instance in astronomy (e.g. the Outer Solar System over 100 million years $[1$, Sect. I.2.4]), or in molecular dynamics [13, Chap.11]. The situation for the Martinet case is quite different because we are interested in the numerical integration of Hamiltonian systems on relatively short time intervals. Nevertheless, symplectic integrators still reveal very efficient. Here, the essential difficulty is that the solution approaches a few times the critical point $\left(y, p_{y}\right)=0$ in the phase space. We show in this section that symplectic integrators resolve close approaches to such a critical point with high accuracy even for large step sizes, whereas non-symplectic ones do not reproduce the correct behavior (except for very small step sizes).

4.2.1. Martinet flat case Consider the Martinet problem (2) in the flat case $\beta=0$. Its interesting dynamics takes place in the $\left(y, p_{y}\right)$ plane, and it is not influenced by the other variables (only by their initial values). We put $\eta=\left(y, p_{y}\right)$, and we denote by $f(\eta)$ the Hamiltonian vector field composed by the corresponding two equations of (2). For a numerical integrator of order $p=2$, the associated modified differential equation has the form

$$
\dot{\tilde{\eta}}=f(\widetilde{\eta})+h^{2} f_{3}(\widetilde{\eta})+\mathcal{O}\left(h^{3}\right) .
$$

Consider first the symplectic Störmer-Verlet method. It follows from Sect.4.1 that its modified differential equation is Hamiltonian, and from (9) that the modified Hamiltonian $\widetilde{H}(\eta)$ is preserved up to exponentially small terms along the numerical solution. This implies that the numerical solution remains exponentially close to a periodic orbit in the $\left(y, p_{y}\right)$-space. The critical point $\left(y=0, p_{y}=0\right)$ is a saddle point also for the modified differential equation (because the origin is stationary also for the numerical solution and thus for the modified equation). Therefore, any numerical solution starting close to the origin has to come back to it after turning around one of the stationary points. The minimal distance to the origin will always stay the same (see the zoom in Figure 2). This explains the good behavior of symplectic integrators.

For the non-symplectic integrator, the term $h^{2} f_{3}(\eta)$ is not Hamiltonian. Therefore the solution of the modified differential equation (and hence also the numerical solution) is no longer periodic. In fact, it spirals outwards and after surrounding the first stationary point, the numerical solution does not approach the saddle point sufficiently close, which induces a faster dynamics as can be observed in Figures 1 and 2. This causes a huge error, because close to the saddle point the numerical solution is most sensible to errors.

4.2.2. Non integrable perturbation In this case, the argument in the comparison of symplectic and non-symplectic integrators is very similar to the discussion of the Van der Pol's equation in $[1$, Sect.XII.1]. For $\beta \neq 0$ (non integrable perturbation), the dynamics takes place in the four dimensional space with variables $\eta=\left(x, y, p_{x}, p_{y}\right)$. In this space the system (2) becomes

$$
\dot{\eta}=f(\eta)+\beta g(\eta)
$$

where $f(\eta)$ is the Hamiltonian vector field corresponding to $\beta=0$ and $g(\eta)=\mathcal{O}(1)$ depends smoothly on $\beta$. Here, the modified equation becomes

$$
\dot{\tilde{\eta}}=f(\widetilde{\eta})+\beta g(\widetilde{\eta})+h^{2} f_{3}(\widetilde{\eta})+\mathcal{O}\left(h^{3}+\beta h^{2}\right),
$$


where the perturbation term $h^{2} f_{3}(\eta)$ is the same as for the Martinet flat case.

For the symplectic integrator, the perturbation $\beta g(\eta)$ has the same effect for the original problem as for $\dot{\tilde{\eta}}=f(\widetilde{\eta})+h^{2} f_{3}(\widetilde{\eta})+\ldots$ This explains the correct qualitative behavior for small $h$ and small $\beta$. There is no restriction on the step size $h$ compared to the size of $\beta$.

For the non-symplectic integrator, each of the perturbation terms $\beta g(\eta)$ and $h^{2} f_{3}(\eta)$ destroys the periodic orbits in the subsystem for the $\left(y, p_{y}\right)$ variables, and the dominant one will determine the behavior of the numerical solution. Only when $h^{2} \ll|\beta|$, the numerical solution will catch the correct dynamics of the problem. In Figures 3 and 4 , where $\beta=-10^{-4}$, this condition is not satisfied for $h \geq 10^{-2}$. Since $\beta$ is chosen small and negative, the two perturbation terms are conflicting. The term $\beta g(\eta)$ causes the solution to spiral around the positive stationary point, whereas the term $h^{2} f_{3}(\eta)$ causes it to spiral alternatively around both stationary points. For too large step sizes the qualitative behavior of the non-symplectic integrator (4) is thus completely wrong.

\section{Remark}

The problem (2) with $\beta=0$ has a lot of symmetries. In the $\left(y, p_{y}\right)$-space the orbits are symmetric with respect to the $y$-axis and also with respect to the $p_{y}$-axis. If we apply a symmetric numerical integrator (not necessarily symplectic), it is possible to prove the same qualitative behavior as for the symplectic Störmer-Verlet method. This follows from the fact that the solution of the modified equation (numerical orbit) corresponding to a symmetric method has the same symmetry properties as the exact flow (see [1, Sect. IX.2] for precise statements). Consequently, in the $\left(y, p_{y}\right)$ plane and for $\beta=0$, the solution will stay exponentially near to a closed orbit, as it is the case for symplectic integrators. In the non integrable case, the good behavior of symmetric methods can be explained as in Sect. 4.2.2 for symplectic methods.

\section{ORBITAL TRANSFER OF A SPACECRAFT}

We consider the orbit transfer problem presented in [14, pp. 66-68] and studied in [6]. The problem is to transfer a spacecraft with constant thrust force $T$ from a given initial circular orbit $r_{0}$ to the largest possible circular orbit for a given length of time $t_{f}$. The control function is the thrust-direction given by an angle $\phi(t)$. The state functions are $(r, u, v)$, where $r(t)$ is the radial distance of spacecraft from attracting center, $u(t)$ is the radial component of velocity, and $v(t)$ is the tangential component of velocity.

The optimal control problem can be formulated as maximizing the radial distance $r\left(t_{f}\right)$ at the final time, subject to the differential equations

$$
\begin{aligned}
\dot{r} & =u \\
\dot{u} & =\frac{v^{2}}{r}-\frac{\mu}{r^{2}}+\frac{T \sin \phi}{m_{0}-|\dot{m}| t} \\
\dot{v} & =-\frac{u v}{r}+\frac{T \cos \phi}{m_{0}-|\dot{m}| t}
\end{aligned}
$$



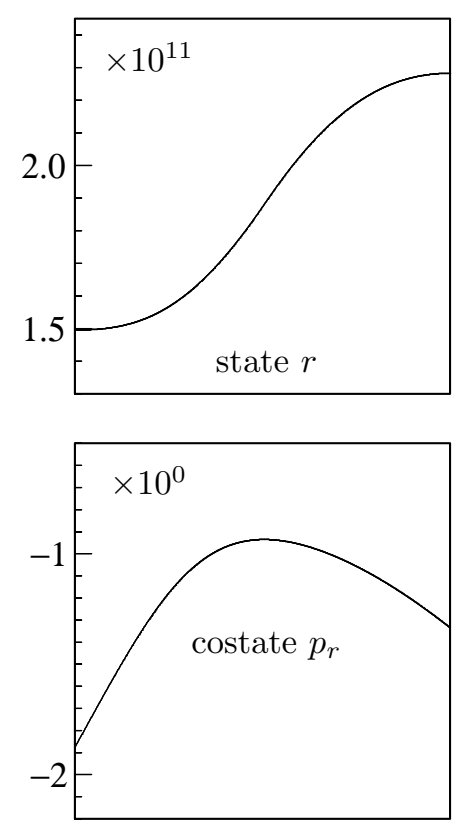
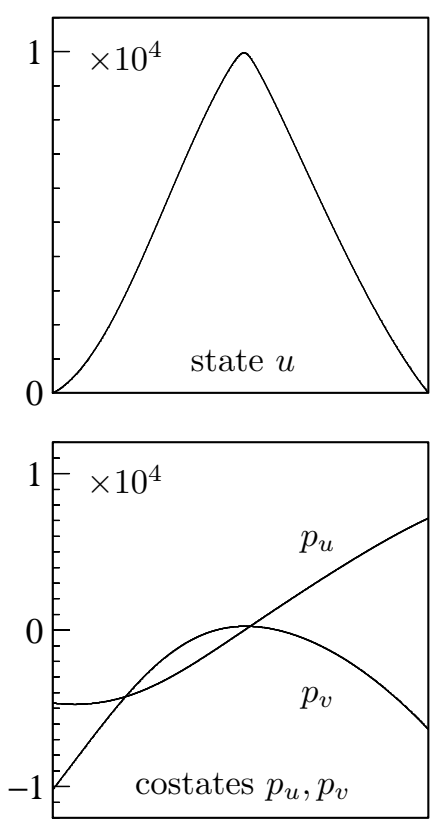
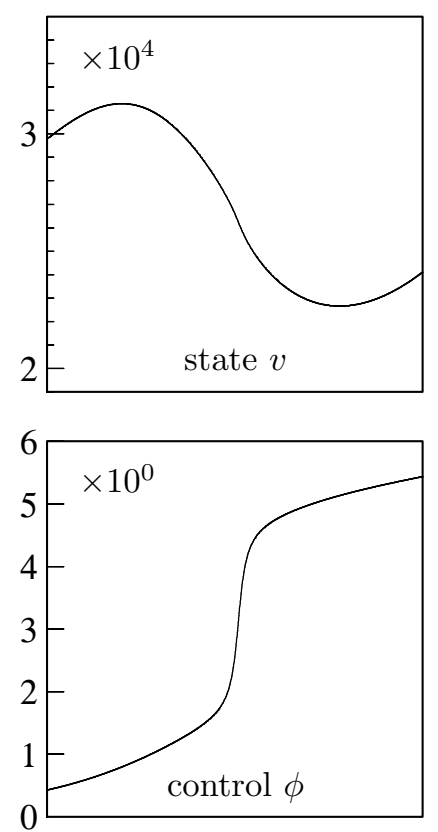

Figure 6. Exact solution of the orbit transfer problem on the time interval $\left[0, t_{f}\right]$.

with boundary conditions

$$
\begin{array}{rl}
r(0)=r_{0}, & u\left(t_{f}\right)=0 \\
u(0)=0 & v\left(t_{f}\right)^{2} r\left(t_{f}\right)-\mu=0 \\
v(0)=\sqrt{\frac{\mu}{r_{0}}} &
\end{array}
$$

The constants are $m_{0}=10000 \mathrm{~kg}$ (initial mass of spacecraft), $|\dot{m}|=12.9 \mathrm{~kg} /$ day (fuel consumption rate), $r_{0}=1.496 \cdot 10^{11} \mathrm{~m}$ (distance Sun-Earth), $T=8.336 \mathrm{~N}$ (thrust force), $\mu=1.32733 \cdot 10^{20} \mathrm{~m}^{3} / \mathrm{s}^{2}$ (gravitational constant for sun), and $t_{f}=193$ days $\left(\approx 1.67 \cdot 10^{7}\right.$ seconds).

We solve this problem using Pontryagin's maximum principle. The Hamiltonian is

$$
H=p_{r} u+p_{u}\left(\frac{v^{2}}{r}-\frac{\mu}{r^{2}}+\frac{T \sin \phi}{m_{0}-|\dot{m}| t}\right)+p_{v}\left(-\frac{u v}{r}+\frac{T \cos \phi}{m_{0}-|\dot{m}| t}\right)+p_{t}
$$

and the differential equation for the adjoint state $\left(p_{r}, p_{u}, p_{v}, p_{t}\right)$ is

$$
\begin{aligned}
\dot{p}_{r} & =p_{u}\left(\frac{v^{2}}{r^{2}}-2 \frac{\mu}{r^{3}}\right)-p_{v} \frac{u v}{r^{2}} \\
\dot{p}_{u} & =-p_{r}+p_{v} \frac{v}{r} \\
\dot{p}_{v} & =-2 p_{u} \frac{v}{r}+p_{v} \frac{u}{r} \\
\dot{p}_{t} & =-p_{u} \frac{T|\dot{m}|}{\left(m_{0}-|\dot{m}| t\right)^{2}} \sin \phi-p_{u} \frac{T|\dot{m}|}{\left(m_{0}-|\dot{m}| t\right)^{2}} \cos \phi, \quad p_{t}(0)=0 .
\end{aligned}
$$

Copyright (c) 2002 John Wiley \& Sons, Ltd. Prepared using ocaauth.cls 
The extremality condition for $p\left(t_{f}\right)$ is given by

$$
p_{v}\left(t_{f}\right) v\left(t_{f}\right)-2\left(p_{r}\left(t_{f}\right)-1\right) r\left(t_{f}\right)=0 .
$$

Applying the Pontryagin principle, the control $\phi(t)$ minimizes the Hamiltonian (13) at all times. This yields

$$
\sin (\phi)=\frac{-p_{u}}{\sqrt{p_{u}^{2}+p_{v}^{2}}}, \quad \cos (\phi)=\frac{-p_{v}}{\sqrt{p_{u}^{2}+p_{v}^{2}}} .
$$

The Hamiltonian system (11) \& (14) with boundary conditions (12) \& (15) is solved by the standard single shooting technique. In Figure 6 we plot the exact solution (computed numerically with high precision). The thrust direction $\phi$ starts close to the tangential direction, and rotates during the orbit transfer with an angle of about $\approx 2 \pi$. At the middle of the time interval, the thrust direction rotates more rapidly.
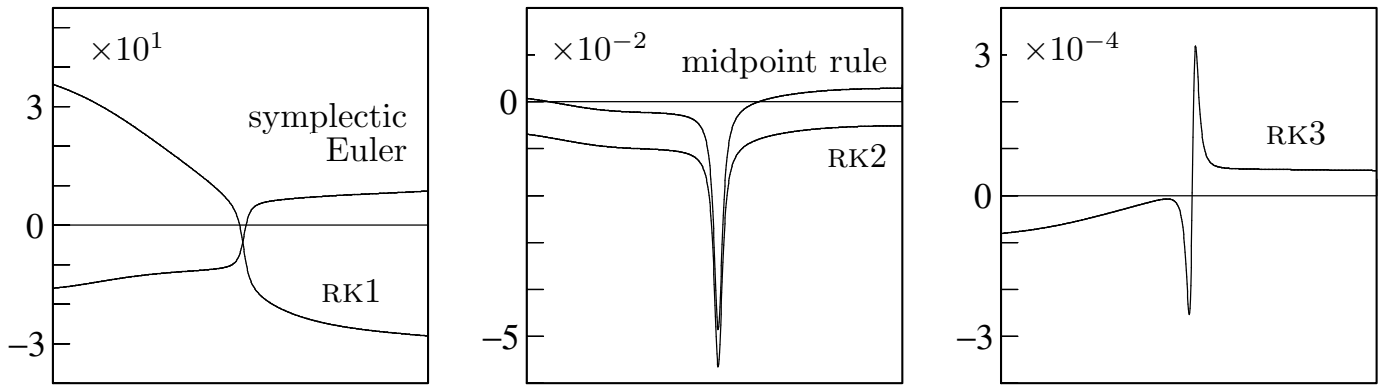

Figure 7. Errors in the Hamiltonian for various numerical integrators applied with constant step size (1000 steps). Explicit Runge Kutta methods (non-symplectic) of orders 1, 2, 3 (RK1,RK2,RK3) compared to the symplectic Euler method (order 1) and the implicit midpoint rule (order 2, symplectic).

In Figure 7 we plot the relative errors in the Hamiltonian as functions of time (compared to the Hamiltonian of the exact solution) for various symplectic and non-symplectic numerical integrators of orders 1, 2, and 3. We do not oberve any significant advantage for the symplectic integrators. We notice a qualitative different behavior of the methods of order 2. There, the error in the Hamiltonian has a peak in the middle of the integration interval, and it comes back to about the same value it had before. For the midpoint rule this is due to its symmetry, and for the second order Runge-Kutta methods this follows from the fact that for methods with even order the dominant error term behaves like that of a symmetric integrator (backward error analysis). The results of this experiment are not really surprising, because the solution does not have a quasi-oscillatory behavior, so that errors in the Hamiltonian could be compensated by a symplectic integrator.

\section{SUBMERGED RIGID BODY}

We consider the autonomous submarine model introduced in [15]. For simplicity, we restrict ourself to the vertical planar situation: the rigid body moves in the $x z$-plane exclusively. The state of the rigid body is given by $q(t)=\left(b_{1}(t), b_{3}(t), \theta(t), \nu_{1}(t), \nu_{3}(t), \Omega_{2}(t)\right)$, where $b_{1}(t), b_{3}(t)$ 
denote the position vector and $\theta(t)$ represents the diving angle, and $\nu_{1}(t), \nu_{3}(t)$, and $\Omega_{2}(t)$ are the corresponding translational and angular velocities.

Given a fixed time interval of length $t_{f}>0$, we search for the energy minimizing trajectory to get the submarine from a configuration $q(0)$ to a configuration $q\left(t_{f}\right)$, e.g. $t_{f}=5$ and

$$
q(0)=(0,0,0,0,0,0), \quad q(5)=(1,1,0,0,0,0) .
$$

Here, the energy is defined as (a more realistic energy model is derived in [16])

$$
E(q)=\frac{1}{2} \int_{0}^{t_{f}}\left(\varphi_{\nu_{1}}^{2}+\varphi_{\nu_{3}}^{2}+\tau_{\Omega_{2}}^{2}\right) d t
$$

where $\varphi_{\nu_{1}}(t), \varphi_{\nu_{3}}(t), \tau_{\Omega_{2}}(t)$ are the control functions. The dynamics are

$$
\begin{aligned}
\dot{b}_{1} & =\nu_{1} \cos \theta+\nu_{3} \sin \theta, \quad \dot{\nu}_{1}=\frac{1}{m_{1}}\left(-m_{3} \nu_{3} \Omega_{2}-D_{\nu}^{2} \nu_{1}^{3}-D_{\nu}^{1} \nu_{1}+G \sin \theta+\varphi_{\nu_{1}}\right) \\
\dot{b}_{3} & =-\nu_{1} \sin \theta+\nu_{3} \cos \theta, \quad \dot{\nu}_{3}=\frac{1}{m_{3}}\left(m_{1} \nu_{1} \Omega_{2}-D_{\nu}^{2} \nu_{3}^{3}-D_{\nu}^{1} \nu_{3}-G \cos \theta+\varphi_{\nu_{3}}\right) \\
\dot{\theta} & =\Omega_{2}, \quad \dot{\Omega}_{2}=\frac{1}{I_{b_{2}}}\left(-D_{\Omega}^{2} \Omega_{2}^{3}-D_{\Omega}^{1} \Omega_{2}+\rho g \mathcal{V}\left(-z_{B} \sin \theta+x_{B} \cos \theta\right)+\tau_{\Omega_{2}}\right)
\end{aligned}
$$

with positive constants: $m_{1}=m_{3}=m+M$ (masses) $m=126.55 \mathrm{~kg}, M=70 \mathrm{~kg}, D_{\nu}^{1}=$ $-27.0273, D_{\nu}^{2}=-897.6553, D_{\Omega}^{1}=-13.793, D_{\Omega}^{2}=-6.45936$ (drags), $G=-3 \mathrm{~N}$ (Archimède), $I_{b_{2}}=5.29 \mathrm{~kg} \cdot \mathrm{m}^{2}, g=9.80 \mathrm{~m} \cdot \mathrm{s}^{-2}, \rho g \mathcal{V}=m g-G, z_{B}=-7 \cdot 10^{-3} \mathrm{~m}, x_{B}=0 \mathrm{~m}$ (buoyancy). These numerical values were derived from experiments performed on a test-bed vehicule, see [15].

Here, the Hamiltonian system is very sensitive when considered as an initial value problem (i.e. $q(0)$ and $p(0)$ given). When ones slightly perturbates initial conditions (e.g. by multiplying $p(0)$ or $q(0)$ by $\left.1+10^{-10}\right)$, the corresponding solution explodes. For this reason, single shooting methods fail to solve the boundary value problem, and we use a multiple shooting method.

For this system we found one normal extremal with a conjugate point. The corresponding states, costates and controls are represented in Figure 8 (using a high-order integrator).
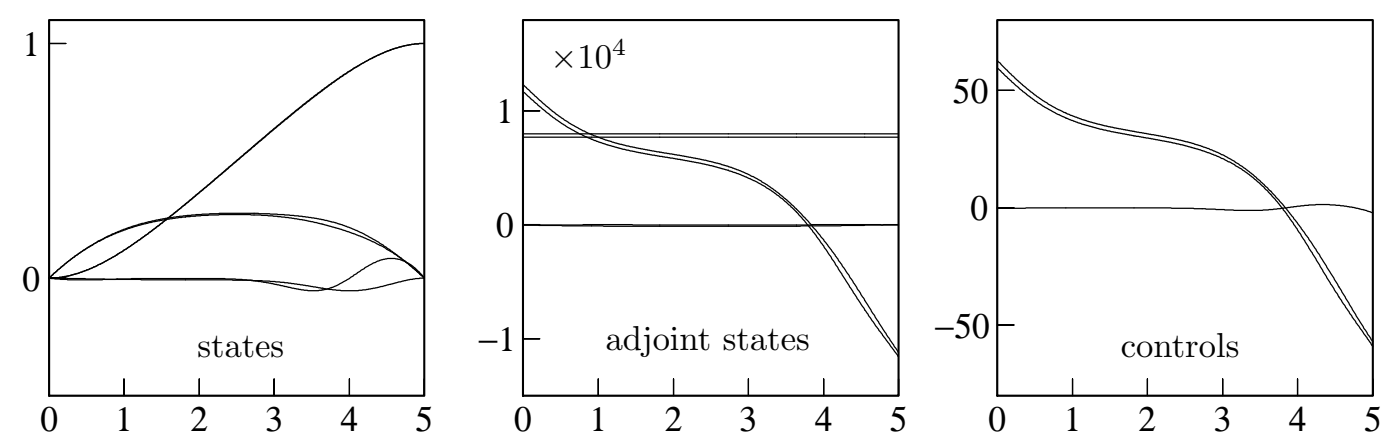

Figure 8. Extremal obtained for $p(0)=(7709.864500233298,7988.036994413952$, $-3.0163588901640024,11707.858394005056,12318.149504556683,-1.0570538454444238)$.

In Figure 9, we compare for the same stepsize $(h=0.05)$ the accuracy of the implicit midpoint rule (order 2), which is a symplectic integrator, with an explicit Runge-Kutta method 

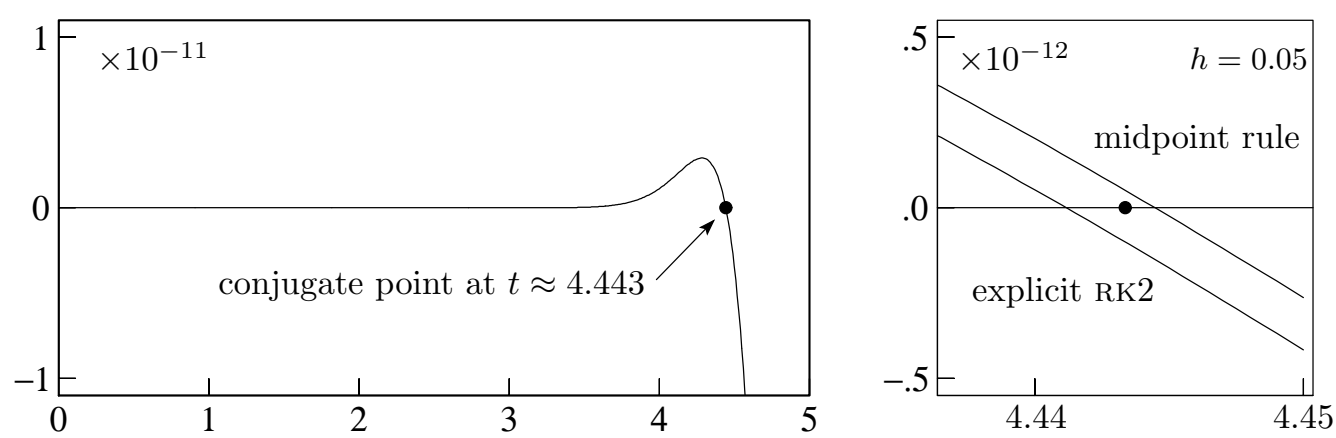

Figure 9. Computation of $\operatorname{det} \frac{\partial q}{\partial p_{0}}$. Left picture: exact solution on the time interval $[0,5]$. Right picture: Implicit midpoint rule (symplectic) and an explicit Runge-Kutta method (RK2, non-symplectic) for the same stepsize $h=0.05$ with cubic Hermite interpolation.

(non symplectic) of the same order (RK2, see (4)). The numerical solution and the determinant of the Jacobian $\frac{\partial q}{\partial p_{0}}$ are obtained on the grid points of integration. For the computation of conjugate points we need a continuous approximation of the solution, which is obtained by cubic Hermite interpolation. The resulting interpolation error is of size $\mathcal{O}\left(h^{4}\right)$ and thus negligible for second order methods. Notice that linear interpolation would introduce an error $\mathcal{O}\left(h^{2}\right)$ that is of the same size as the truncation (global) error of the numerical integrators. The mark in the middle of Figure 9 corresponds to the conjugate point of the exact solution. There is again no real advantage for the symplectic integrator. The implicit midpoint rule (symplectic) is only twice more accurate than the non-symplectic method RK2, and this is due to the size of the error constants of the methods and not to symplecticity.

\section{CONCLUSION}

From the point of view of "geometric numerical integration" it is natural to use symplectic integrators when solving Hamiltonian differential equations. This has been proved very successfully in many fields, in particular, in molecular dynamics simulations and in longtime integrations of planetary motion. For Hamiltonian systems arising form the Pontryagin maximum principle in optimal control our conclusion is the following.

Due to the fact that one is concerned with boundary value problems, long-term integration is not an issue. For integrations over short intervals, e.g. half a period of the motion of a planet, there is no real advantage of using a symplectic method. The solutions of problems of Sects. 5 and 6 neither show a periodic or quasi-periodic behavior nor an ergodic behavior like in molecular dynamics simulations. Therefore, no improvement of symplectic integrators can be expected, which is confirmed by numerical experiments.

For special problems, typically in low dimension and in situations where the Hamiltonian is close to a critical value (Sect. 3), the structure preservation of symplectic integrators is very important and symplectic methods can be much more efficient than non-symplectic ones. 
Indeed, the theory of backward error analysis allows one to prove that the numerical solution of a symplectic method has the same qualitative behavior as the exact flow. For example, in the integrable Martinet case, where the exact solution is periodic, the numerical solution remains exponentially close to a periodic orbit, which explains the excellent results, even on a relatively short interval of integration (a few periods). For non symplectic integrators, this structure is destroyed in general.

\section{REFERENCES}

1. E. Hairer, C. Lubich, and G. Wanner. Geometric Numerical Integration. Structure-Preserving Algorithms for Ordinary Differential Equations. Springer Series in Computational Mathematics 31, Second Edition, Springer-Verlag, Berlin, 2006.

2. J.M. Wendlandt and J. E. Marsden, Mechanical integrators derived from a discrete variational principle, Physica D 106, 223-246, 1997.

3. J. E. Marsden and M. West, Discrete mechanics and variational integrators Acta Numerica 10, 357-514, 2001 .

4. A. M. Bloch, P. E. Crouch, J. E. Marsden, and T. S. Ratiu, The symmetric representation of the rigid body equations and their discretization, Nonlinearity vol. 15 pp. 1309-1341, 2002.

5. V.M. Guibout and A. Bloch. Discrete variational principles and Hamilton-Jacobi theory for mechanical systems and optimal control theory, to appear, 2004. http://hal.archives-ouvertes.fr/hal-00002863/en/

6. W. Hager. Runge-Kutta methods in optimal control and the transformed adjoint system Numer. Math. $87(2), 247-282,2000$.

7. J. F. Bonnans and J. Laurent-Varin. Computation of order conditions for symplectic partitioned RungeKutta schemes with application to optimal control Numer. Math. 103(1), 1-10, 2006.

8. A.A. Agrachev, B. Bonnard, M. Chyba, and I. Kupka. Sub-Riemannian sphere in Martinet flat case. ESAIM/COCV (Control, Optimisation and Calculus of Variations), pp. 377-448, 1997.

9. B. Bonnard, M. Chyba, and I. Kupka. Non Integrable Geodesics in SR-Martinet Geometry. Proceedings of the 1997 Summer Research Institute on Differential Geometry and Control, pp. 119-13, 1999.

10. B. Bonnard, G. Launay, and E. Trelat. The transcendence needed to compute the sphere and wave front in Martinet SR-geometry. J. Math. Sci., New York 103, 6, pp. 688-708, 2001.

11. B. Bonnard and E. Trelat. On the role of abnormal minimizers in SR-geometry. Ann. Fac. Sci. Toulouse 6, X, 3, pp. 405-491, 2001

12. J. M. Sanz-Serna and M. P. Calvo. Numerical Hamiltonian Problems. Chapman E Hall, London, 1994.

13. B. Leimkuhler and S. Reich. Simulating Hamiltonian Dynamics. Cambridge Monographs on Applied and Computational Mathematics 14, Cambridge University Press, Cambridge, 2004.

14. A. E. Bryson, Jr., Y.-C. Ho. Applied Optimal Control. Blaisdell, Waltham, MA, 1969.

15. M. Chyba, T. Haberkorn, R.N. Smith and S.K. Choi. Design and Implementation of Time Efficient Trajectories for Underwater vehicles. Journal of Ocean Engineering. To appear, 2008. (doi:10.1016/j.oceaneng.2007.07.007)

16. M. Chyba, T. Haberkorn, R.N. Smith and S.K. Choi. Autonomous Underwater vehicles: Development and Implementation of Time and Energy Efficient Trajectories. Ship Technology Research, To appear, 2008. 